\title{
Eisenstein series and their applications to some arithmetic identities and congruences
}

Daeyeoul Kim ${ }^{1 *}$, Aeran Kim² and Ayyadurai Sankaranarayanan ${ }^{3,4}$

"Correspondence:

daeyeoul@nims.re.kr

${ }^{1}$ National Institute for Mathematical Sciences, Yuseong-daero 1689-gil,

Yuseong-gu, Daejeon, 305-811, South Korea

Full list of author information is available at the end of the article

\begin{abstract}
Utilizing the theory of elliptic curves over $\mathbb{C}$ to the normalized lattice $\Lambda_{\tau}$, its connection to the Weierstrass $\wp$-functions and to the Eisenstein series $E_{4}(\tau)$ and $E_{6}(\tau)$, we establish some arithmetic identities involving certain arithmetic functions and convolution sums of restricted divisor functions. We also prove some congruence relations involving certain divisor functions and restricted divisor functions.

MSC: Primary 11A25; secondary 11A07; 11G99
\end{abstract}

Keywords: divisor functions; restricted divisor functions; $q$-series; $q$-products; Eisenstein series; convolution sums; congruence relations

\section{Introduction}

The study of arithmetical identities and congruences is classical in number theory and such investigations have been carried out by several mathematicians including the legend Srinivasa Ramanujan. This study constitutes an important and significant part of the subject number theory.

For $N, m, r, s, d \in \mathbb{Z}$ with $d, s>0$ and $r \geq 0$, we define some divisor functions for our use in the sequel. Let

$$
\sigma_{s}(N)=\sum_{d \mid N} d^{s}
$$

and let us define the restricted divisor function

$$
\sigma_{s, r}(N ; m)=\sum_{\substack{d \mid N \\ d \equiv r \bmod m}} d^{s} .
$$

Note that

$$
\sigma(N):=\sigma_{1}(N)=\sum_{d \mid N} d
$$

For $a, b, n \in \mathbb{N}$, let us define the convolution sum

$$
S_{a, b}(n):=\sum_{m=1}^{n-1} \sigma_{a}(m) \sigma_{b}(n-m) .
$$

(c) 2013 Kim et al.; licensee Springer. This is an Open Access article distributed under the terms of the Creative Commons Attribution License (http://creativecommons.org/licenses/by/2.0), which permits unrestricted use, distribution, and reproduction in any medium, provided the original work is properly cited. 
Ramanujan showed that the sum $S_{a, b}(n)$ can be evaluated in terms of $\sigma_{a+b+1}(n), \sigma_{a+b-1}(n)$, $\ldots, \sigma_{3}(n), \sigma_{1}(n)$ for the nine pairs $(a, b) \in \mathbb{N}^{2}$ satisfying $a+b=2,4,6,8,12, a \leq b, a \equiv b \equiv 1$ (mod 2). For example, explicitly, we know (see [1]) that

$$
S_{1,11}(n)=\frac{691}{65,520} \sigma_{13}(n)+\left(\frac{1}{24}-\frac{1}{24} n\right) \sigma_{11}(n)-\frac{691}{65,520} \sigma_{1}(n)
$$

and (see [2, p.35])

$$
S_{3,9}(n)=\frac{1}{2,640} \sigma_{13}(n)-\frac{1}{240} \sigma_{9}(n)+\frac{1}{264} \sigma_{3}(n) .
$$

From [3], we note that for any integer $n \geq 3$, we have

$$
\begin{aligned}
& \sum_{\substack{\left(m_{1}, m_{2}, m_{3}\right) \in \mathbb{N}^{3} \\
m_{1}+m_{2}+m_{3}=n}} m_{1} m_{2} \sigma_{1}\left(m_{1}\right) \sigma_{1}\left(m_{2}\right) \sigma_{1}\left(m_{3}\right) \\
& =\frac{1}{288}\left(n^{2} \sigma_{5}(n)+\left(n^{2}-4 n^{3}\right) \sigma_{3}(n)-\left(n^{3}-3 n^{4}\right) \sigma_{1}(n)\right) .
\end{aligned}
$$

For an elementary proof of (1) and (2), we refer to [4]. An another interesting arithmetical identity (which was stated by Ramanujan, see [1, p.146], for some analytical proofs of this identity, one may refer to [5, p.329], [6, p.136] and [7], also [4]) is for $n \in \mathbb{N}$, we have

$$
\sum_{k=0}^{n-1} \sigma_{1}(2 k+1) \sigma_{3}(n-k)=\frac{1}{240} \sigma_{5}(2 n+1)-\frac{1}{240} \sigma_{3}(2 n+1) .
$$

There are some nice arithmetical identities connecting the divisor functions along with Ramanujan's $\tau$-function. For instance, we know (see [8]) that

$$
\sum_{m=1}^{n-1} m(n-m) \sigma_{3}(m) \sigma_{3}(n-m)=\frac{1}{540} n^{2} \sigma_{7}(n)-\frac{1}{540} \tau(n)
$$

and from [9], we observe that for $n \in \mathbb{N} \cup\{0\}$,

$$
t_{24}(n)=\frac{1}{176,896}\left(\sigma_{11}^{*}(n+3)-\tau(n+3)-2,072 \tau\left(\frac{n+3}{2}\right)\right),
$$

where $t_{k}(n)$ denotes the number of representations of $n$ as a sum of $k$ triangular numbers, i.e. (with $\mathbb{N}_{0}=\mathbb{N} \cup\{0\}$ ),

$$
\begin{aligned}
& t_{k}(n):= \#\left\{\left(m_{1}, \ldots, m_{k}\right) \in \mathbb{N}_{0}^{k} \mid n=\frac{1}{2} m_{1}\left(m_{1}+1\right)+\cdots+\frac{1}{2} m_{k}\left(m_{k}+1\right)\right\}, \\
& \sigma_{k}^{*}(n):=\sum_{\substack{d \mid n \\
\frac{n}{d} \text { odd }}} d^{k},
\end{aligned}
$$

and $\tau(n)$ is the coefficient defined by the expression:

$$
q\left\{(1-q)\left(1-q^{2}\right) \cdots\right\}^{24}=\sum_{n=1}^{\infty} \tau(n) q^{n}, \quad q \in \mathbb{C} \text { with }|q|<1 .
$$


For any integer $k \geq 2$, let

$$
r_{k}(n):=\#\left\{\left(x_{1}, \ldots, x_{k}\right) \in \mathbb{Z}^{k} \mid n=x_{1}^{2}+\cdots+x_{k}^{2}\right\} .
$$

An another identity worth mentioning (see [1]) is

$$
r_{24}(n)=\frac{16}{691} \sigma_{11}^{* * *}(n)+\frac{128}{691}\left(259(-1)^{n-1} \tau(n)-512 \tau\left(\frac{n}{2}\right)\right),
$$

where $\sigma_{k}^{* * *}(n):=\sum_{d \mid n}(-1)^{d} d^{k}$ and $\tau\left(\frac{n}{2}\right)=0$ if $\frac{n}{2}$ is not an integer.

From (3), (4) and (5), it is immediate (and interesting) to note that, for $n \in \mathbb{N}$,

$$
\begin{aligned}
& n^{2} \sigma_{5}(n)+\left(n^{2}-4 n^{3}\right) \sigma_{3}(n)-\left(n^{3}-3 n^{4}\right) \sigma_{1}(n) \equiv 0 \quad(\bmod 288) \\
& \sigma_{5}(2 n+1)-\sigma_{1}(2 n+1) \equiv 0 \quad(\bmod 240)
\end{aligned}
$$

and

$$
n^{2} \sigma_{7}(n)-\tau(n) \equiv 0 \quad(\bmod 540)
$$

The proofs of all these identities and congruences heavily depend upon the theory of modular functions and the properties of Eisenstein series. Later some of these identities have been proved using only elementary techniques.

Define the integers $a(n)$ (for $n \in \mathbb{N}$ ) by

$$
\sum_{n=1}^{\infty} a(n) q^{n}=q \prod_{n=1}^{\infty}\left(1-q^{2 n}\right)^{12}, \quad q \in \mathbb{C} \text { with }|q|<1 .
$$

Also define the integers $b(n)$ (for $n \in \mathbb{N}$ ) by

$$
\sum_{n=1}^{\infty} b(n) q^{n}=q \prod_{n=1}^{\infty}\left(1-q^{2 n}\right)^{4}\left(1-q^{4 n}\right)^{4}, \quad q \in \mathbb{C} \text { with }|q|<1
$$

Note that $a(n) \equiv 0$ if $n \equiv 0(\bmod 2)$ and $b(n) \equiv 0$ if $n \equiv 0(\bmod 2)$. It has been proved by Kenneth Williams (see $[10,11])$ that $($ for $n \in \mathbb{N})$,

$$
r_{12}(n)=8 \sigma_{5}(n)-512 \sigma_{5}\left(\frac{n}{4}\right)+16 a(n)
$$

and

$$
\begin{aligned}
A_{8}(n)= & \frac{1}{192} \sigma_{3}(n)+\frac{1}{64} \sigma_{3}\left(\frac{n}{2}\right)+\frac{1}{16} \sigma_{3}\left(\frac{n}{4}\right)+\frac{1}{3} \sigma_{3}\left(\frac{n}{8}\right) \\
& +\left(\frac{1}{24}-\frac{1}{32} n\right) \sigma_{1}(n)+\left(\frac{1}{24}-\frac{1}{4} n\right) \sigma_{1}\left(\frac{n}{8}\right)-\frac{1}{64} b(n),
\end{aligned}
$$

where $r_{12}(n)$ is as mentioned before and for $s, n \in \mathbb{N}$,

$$
A_{s}(n):=\sum_{\substack{k \in \mathbb{N} \\ k<\frac{n}{s}}} \sigma_{1}(k) \sigma_{1}(n-s k)
$$


It should be mentioned that Bernoulli identities associated with the Weierstrass $\wp$-function have been studied by Chang and Srivastava in [12]. Families of Weierstrass type functions and their applications have been investigated by Chang, Srivastava and Wu in [13]. It is also interesting to note that the families of Weierstrass type functions, Weber type functions and their applications have been studied by Aygunes and Simsek in [14]. A few more related references are [15, 16] and [17].

Though there are plenty of identities and congruences involving various arithmetic functions available in the literature, practically nothing seriously known involving restricted divisor functions.

For any integer $M \geq 2$ with $M=p_{1}^{e_{1}} \cdots p_{r}^{e_{r}}$, we define $\operatorname{ord}_{p_{j}} M:=e_{j}$.

Throughout the paper, $q=e^{2 \pi i \tau}$ where $\tau \in \mathfrak{h}=\{x+y i \mid y>0\}$ unless otherwise specified hereafter. The aim of this article is to prove some arithmetical identities involving certain arithmetic functions and convolution sums of restricted divisor functions. We also establish some congruence relations similar to (8), (9) and (10). More precisely, we prove the following theorems.

\section{Theorem 1.1}

(i) For any integer $M \geq 2$, we have

$$
11 \sigma_{3}(M) \equiv \sigma_{3}(2 M)+2 \sigma_{1,1}(M ; 2) \quad(\bmod 24)
$$

(ii) Moreover, if $M$ is odd or $\operatorname{ord}_{p} M$ is odd for an odd prime $p$, then

$$
11 \sigma_{3}(M) \equiv \sigma_{3}(2 M)+2 \sigma_{1,1}(M ; 2) \quad(\bmod 48)
$$

Theorem 1.2 For any integer $M \geq 3$, we have

$$
-7 \sigma_{5}(M)+\sigma_{1,1}(M ; 2) \equiv 12 \sigma_{3}(M)-2 \sigma_{3}(2 M) \quad(\bmod 192) .
$$

Theorem 1.3 Let $N \in \mathbb{N}$. Then, we have

$$
\begin{aligned}
& \sum_{\substack{n+l+m-1=N \\
n, l, m \geq 1}} \sigma_{1,1}(2 n-1 ; 2) \sigma_{1,1}(2 l-1 ; 2) \sigma_{1,1}(2 m-1 ; 2) \\
& =\frac{1}{256}\left[\sigma_{5}(2 N-1)-a(2 N-1)\right]
\end{aligned}
$$

where

$$
\sum_{N=1}^{\infty} a(N) q^{N}=q \prod_{N=1}^{\infty}\left(1-q^{2 N}\right)^{12}
$$

Corollary 1.4 For $N \in \mathbb{N}$, we have

$$
(N+1) a(2 N-1) \equiv(N+1) \sigma_{5}(2 N-1) \quad(\bmod 768) .
$$

In particular, if $N \equiv 0,4(\bmod 6)$, then we have

$$
a(2 N-1) \equiv \sigma_{5}(2 N-1) \quad(\bmod 768) .
$$


Theorem 1.5 Let $N \in \mathbb{N}$. Then we have

$$
\begin{aligned}
& \sum_{M=1}^{N-1} \sum_{m=1}^{M-1} \sigma_{1,1}(2 N-1-2 M ; 2) \sigma_{1,1}(M-m ; 2) \sigma_{1,1}(m ; 2) \\
& \quad=\frac{1}{2,304}\left[\sigma_{5}(2 N-1)-8 \sigma_{3}(2 N-1)+4 \sigma_{1}(2 N-1)+3 a(2 N-1)\right] .
\end{aligned}
$$

Remark The main idea in proving Theorems 1.1 and 1.2 is to obtain $q$-series expansions for $E_{4}(\tau)$ and $E_{6}(\tau)$ with coefficients being restricted divisor functions and their convolution sums. Then we have to compare these expressions with the already known $q$-series expressions of $E_{4}(\tau)$ and $E_{6}(\tau)$.

The paper is organized as follows. In Section 2, we express $g_{2}(\tau)$ and $g_{3}(\tau)$ in terms of $q$-products. In Section 3, $g_{2}(\tau)$ and $g_{3}(\tau)$ are transformed into expressions involving $S_{1}$ and $S_{2}$ where $S_{1}$ and $S_{2}$ are $q$-series expressions with coefficients as restricted divisor functions. Then we obtain $q$-series expressions for $E_{4}(\tau)$ and $E_{6}(\tau)$ with coefficients being restricted divisor functions and their convolution sums. Then we compare these expressions of $E_{4}(\tau)$ and $E_{6}(\tau)$ with already known expressions. Section 4 concludes the proofs of Theorems 1.1 and 1.2. In proving arithmetical identities involving the coefficient $a(n)$ (where $a(n)$ is defined as in (11)), we need to study the quantities $S_{1}^{3}$ and $S_{1} S_{2}^{2}$ and in turn the convolution sums of restricted divisor functions along with $a(n)$ come out very naturally.

\section{2 q-product expressions for the Eisenstein series $g_{2}(\tau)$ and $g_{3}(\tau)$}

Let $\Lambda_{\tau}=\mathbb{Z}+\tau \mathbb{Z}(\tau \in \mathfrak{H}$ the complex upper half plane) be a lattice and $z \in \mathbb{C}$. The Weierstrass $\wp$ function relative to $\Lambda_{\tau}$ is defined by the series

$$
\wp\left(z ; \Lambda_{\tau}\right)=\frac{1}{z^{2}}+\sum_{\substack{\omega \in \Lambda_{\tau} \\ \omega \neq 0}}\left\{\frac{1}{(z-\omega)^{2}}-\frac{1}{\omega^{2}}\right\},
$$

and the Eisenstein series of weight $2 k$ for $\Lambda_{\tau}$ with $k>1$ is the series

$$
G_{2 k}\left(\Lambda_{\tau}\right)=\sum_{\substack{\omega \in \Lambda_{\tau} \\ \omega \neq 0}} \omega^{-2 k}
$$

We shall use the notations $\wp(z)$ and $G_{2 k}$ instead of $\wp\left(z ; \Lambda_{\tau}\right)$ and $G_{2 k}\left(\Lambda_{\tau}\right)$, respectively, when the lattice $\Lambda_{\tau}$ has been fixed. Then the Laurent series for $\wp(z)$ about $z=0$ is given by

$$
\wp(z)=z^{-2}+\sum_{k=1}^{\infty}(2 k+1) G_{2 k+2} z^{2 k} .
$$

As is customary, by setting

$$
g_{2}(\tau)=g_{2}\left(\Lambda_{\tau}\right)=60 G_{4} \quad \text { and } \quad g_{3}(\tau)=g_{3}\left(\Lambda_{\tau}\right)=140 G_{6} \text {, }
$$

the algebraic relation between $\wp(z)$ and $\wp^{\prime}(z)$ becomes

$$
\wp^{\prime}(z)^{2}=4 \wp(z)^{3}-g_{2}(\tau) \wp(z)-g_{3}(\tau) .
$$


We use the following $q$-product expressions:

$$
\begin{array}{ll}
P_{0}=\prod_{n=1}^{\infty}\left(1-q^{2 n}\right), & P_{1}=\prod_{n=1}^{\infty}\left(1-q^{2 n-1}\right), \\
P_{2}=\prod_{n=1}^{\infty}\left(1+q^{2 n}\right), & P_{3}=\prod_{n=1}^{\infty}\left(1+q^{2 n-1}\right) .
\end{array}
$$

Theorem 2.1 We have

$$
g_{2}(\tau)=\frac{4 \pi^{4}}{3} P_{0}^{8}\left(P_{3}^{16}-2^{4} q P_{2}^{8} P_{3}^{8}+2^{8} q^{2} P_{2}^{16}\right)
$$

and

$$
g_{3}(\tau)=\frac{4 \pi^{6}}{27} P_{0}^{12}\left(-2 P_{3}^{24}+3 P_{1}^{8} P_{3}^{16}+3 P_{1}^{16} P_{3}^{8}-2 P_{1}^{24}\right) .
$$

To prove Theorem 2.1, we need the following lemma.

Lemma 2.2 Let $e_{1}=\wp\left(\frac{\tau}{2}\right), e_{2}=\wp\left(\frac{1}{2}\right)$ and $e_{3}=\wp\left(\frac{\tau+1}{2}\right)$.

(1) $e_{2}-e_{1}=\pi^{2} P_{0}^{4} P_{3}^{8}$.

(2) $e_{2}-e_{3}=\pi^{2} P_{0}^{4} P_{1}^{8}$.

(3) $e_{3}-e_{1}=2^{4} \pi^{2} q P_{0}^{4} P_{2}^{8}$.

Proof See [18].

Proof of Theorem 2.1 From [19, p.63], we observe that $e_{1}, e_{2}$ and $e_{3}$ are the roots of the equation

$$
4 \wp(z)^{3}-g_{2}(\tau) \wp(z)-g_{3}(\tau)=0 .
$$

Therefore, we have

$$
\begin{aligned}
& e_{1}+e_{2}+e_{3}=0, \\
& e_{1} e_{2}+e_{2} e_{3}+e_{3} e_{1}=-\frac{g_{2}(\tau)}{4}
\end{aligned}
$$

and

$$
e_{1} e_{2} e_{3}=\frac{g_{3}(\tau)}{4}
$$

By the above equations and Lemma 2.2, we deduce that

$$
2 e_{1}+e_{3}=e_{1}-\left(-e_{3}-e_{1}\right)=e_{1}-e_{2}=-\pi^{2} P_{0}^{4} P_{3}^{8},
$$


and the following three identities:

$$
\begin{aligned}
e_{1} & =\frac{1}{3}\left[\left(2 e_{1}+e_{3}\right)+\left(e_{1}-e_{3}\right)\right] \\
& =\frac{1}{3}\left(-\pi^{2} P_{0}^{4} P_{3}^{8}-2^{4} \pi^{2} q P_{0}^{4} P_{2}^{8}\right) \\
& =-\frac{\pi^{2}}{3} P_{0}^{4}\left(P_{3}^{8}+2^{4} q P_{2}^{8}\right), \\
e_{3} & =e_{1}+2^{4} \pi^{2} q P_{0}^{4} P_{2}^{8}=-\frac{\pi^{2}}{3} P_{0}^{4}\left(P_{3}^{8}-2^{5} q P_{2}^{8}\right)
\end{aligned}
$$

and

$$
e_{2}=e_{1}+\pi^{2} P_{0}^{4} P_{3}^{8}=-\frac{\pi^{2}}{3} P_{0}^{4}\left(-2 P_{3}^{8}+2^{4} q P_{2}^{8}\right) \text {. }
$$

Using (14), (15) and (16), we obtain the identities for $g_{2}(\tau)$ and $g_{3}(\tau)$, namely

$$
\begin{aligned}
g_{2}(\tau)= & -4\left(e_{1} e_{2}+e_{2} e_{3}+e_{3} e_{1}\right) \\
= & -\frac{4 \pi^{4}}{9} P_{0}^{8}\left[\left(P_{3}^{8}+2^{4} q P_{2}^{8}\right)\left(-2 P_{3}^{8}+2^{4} q P_{2}^{8}\right)\right. \\
& +\left(P_{3}^{8}-2^{5} q P_{2}^{8}\right)\left(-2 P_{3}^{8}+2^{4} q P_{2}^{8}\right) \\
& \left.+\left(P_{3}^{8}+2^{4} q P_{2}^{8}\right)\left(P_{3}^{8}-2^{5} q P_{2}^{8}\right)\right] \\
= & \frac{4 \pi^{4}}{3} P_{0}^{8}\left(P_{3}^{16}-2^{4} q P_{2}^{8} P_{3}^{8}+2^{8} q^{2} P_{2}^{16}\right)
\end{aligned}
$$

and

$$
\begin{aligned}
g_{3}(\tau) & =4 e_{1} e_{2} e_{3} \\
& =-\frac{4 \pi^{6}}{27} P_{0}^{12}\left(P_{3}^{8}+2^{4} q P_{2}^{8}\right)\left(-2 P_{3}^{8}+2^{4} q P_{2}^{8}\right)\left(P_{3}^{8}-2^{5} q P_{2}^{8}\right) \\
& =\frac{4 \pi^{6}}{27} P_{0}^{12}\left(-2 P_{3}^{24}+3 P_{1}^{8} P_{3}^{16}+3 P_{1}^{16} P_{3}^{8}-2 P_{1}^{24}\right)
\end{aligned}
$$

This proves the theorem.

\section{Eisenstein series and divisor functions}

We use the $q$-series and $q$-products notions

$$
\begin{aligned}
& S_{1}:=\sum_{N \text { odd }} \sigma_{1,1}(N ; 2) q^{N}, \\
& S_{2}:=\sum_{N \geq 2 \text { even }} \sigma_{1,1}(N ; 2) q^{N}, \\
& (a ; q)_{\infty}:=(a)_{\infty}:=\prod_{n \geq 0}\left(1-a q^{n}\right)
\end{aligned}
$$

in the following. Some identities of the basic hypergeometric series type are quoted by Fine (see [20]). Some of these identities (in a similar form) can also be found in [21] and [22]. It 
should be mentioned that some generalizations and basic $q$-extensions of Bernoulli, Euler and Genocchi polynomials have been studied recently by Srivastava (see [23]). We also refer to [24] in which zeta and $q$-zeta function, associated series and integrals have been investigated by Srivastava and Choi. We mention below two identities (see [20, p.78, p.79]) for our further use. These are

$$
\frac{\left(q^{2} ; q^{2}\right)_{\infty}^{20}}{(q)_{\infty}^{8}\left(q^{4} ; q^{4}\right)_{\infty}^{8}}=1+8 \sum_{N=1}^{\infty} q^{N}\left(2+(-1)^{N}\right) \sum_{\substack{\omega \mid N \\ \omega \text { odd }}} \omega
$$

and

$$
\frac{q\left(q^{4} ; q^{4}\right)_{\infty}^{8}}{\left(q^{2} ; q^{2}\right)_{\infty}^{4}}=\sum_{N \text { odd }} \sigma(N) q^{N}
$$

Using (19), (20) and the facts,

$$
\prod_{n=1}^{\infty}\left(1-q^{2 n-1}\right)=\prod_{n=1}^{\infty} \frac{\left(1-q^{n}\right)}{\left(1-q^{2 n}\right)}, \quad \prod_{n=1}^{\infty}\left(1+q^{2 n-1}\right)=\prod_{n=1}^{\infty} \frac{\left(1-q^{2 n}\right)}{\left(1-q^{n}\right)} \frac{\left(1-q^{2 n}\right)}{\left(1-q^{4 n}\right)}
$$

and

$$
\prod_{n=1}^{\infty}\left(1+q^{2 n}\right)=\prod_{n=1}^{\infty} \frac{\left(1-q^{4 n}\right)}{\left(1-q^{2 n}\right)},
$$

our aim here is first to prove the following lemma.

Lemma 3.1 Let $q=e^{2 \pi i \tau}, \tau \in \mathfrak{H}$. Then we have

(a)

$$
\wp\left(\frac{\tau}{2}\right)=-\frac{\pi^{2}}{3}\left(1+24 S_{1}+24 S_{2}\right)
$$

(b)

$$
\wp\left(\frac{\tau+1}{2}\right)=-\frac{\pi^{2}}{3}\left(1-24 S_{1}+24 S_{2}\right) .
$$

(c)

$$
\wp\left(\frac{1}{2}\right)=\frac{2 \pi^{2}}{3}\left(1+24 S_{2}\right) .
$$

Proof

(a) From (14), we have

$$
\begin{aligned}
\wp\left(\frac{\tau}{2}\right) & =-\frac{\pi^{2}}{3}\left(\frac{\left(q^{2} ; q^{2}\right)_{\infty}^{20}}{(q)_{\infty}^{8}\left(q^{4} ; q^{4}\right)_{\infty}^{8}}+16 \frac{q\left(q^{4} ; q^{4}\right)_{\infty}^{8}}{\left(q^{2} ; q^{2}\right)_{\infty}^{4}}\right) \\
& =-\frac{\pi^{2}}{3}\left(1+8 \sum_{N=1}^{\infty} q^{N}\left(2+(-1)^{N}\right) \sum_{\substack{\omega \mid N \\
\omega \text { odd }}} \omega+16 \sum_{\text {Nodd }} \sigma(N) q^{N}\right)
\end{aligned}
$$




$$
\begin{aligned}
& =-\frac{\pi^{2}}{3}\left(1+8 \sum_{N \text { odd }} q^{N} \sum_{\substack{\omega \mid N \\
\omega \text { odd }}} \omega+24 \sum_{N \text { even }} q^{N} \sum_{\substack{\omega \mid N \\
\omega \text { odd }}} \omega+16 \sum_{N \text { odd }} \sigma(N) q^{N}\right) \\
& =-\frac{\pi^{2}}{3}\left(1+24 \sum_{N=1}^{\infty} q^{N} \sum_{\substack{\omega \mid N \\
\omega \text { odd }}} \omega\right) \\
& =-\frac{\pi^{2}}{3}\left(1+24 \sum_{N=1}^{\infty} \sigma_{1,1}(N ; 2) q^{N}\right) \\
& =-\frac{\pi^{2}}{3}\left(1+24 S_{1}+24 S_{2}\right) .
\end{aligned}
$$

(b) From (15), we have

$$
\begin{aligned}
& \wp\left(\frac{\tau+1}{2}\right) \\
& =-\frac{\pi^{2}}{3}\left(\frac{\left(q^{2} ; q^{2}\right)_{\infty}^{20}}{(q)_{\infty}^{8}\left(q^{4} ; q^{4}\right)_{\infty}^{8}}-32 \frac{q\left(q^{4} ; q^{4}\right)_{\infty}^{8}}{\left(q^{2} ; q^{2}\right)_{\infty}^{4}}\right) \\
& =-\frac{\pi^{2}}{3}\left(1+8 \sum_{N=1}^{\infty} q^{N}\left(2+(-1)^{N}\right) \sum_{\omega \mid N} \omega-32 \sum_{N \text { odd }} \sigma(N) q^{N}\right) \\
& =-\frac{\pi^{2}}{3}\left(1+8 \sum_{N \text { odd }} q^{N} \sum_{\omega \mid N} \omega+24 \sum_{N \text { even }} q^{N} \sum_{\omega \mid N} \omega-32 \sum_{\omega \text { odd }} \sigma(N) q^{N}\right) \\
& =-\frac{\pi^{2}}{3}\left(1+24 \sum_{N=1}^{\infty}(-1)^{N} q^{N} \sum_{\omega \mid N} \omega\right) \\
& =-\frac{\pi^{2}}{3}\left(1+24 \sum_{N=1}^{\infty}(-1)^{N} \sigma_{1,1}(N ; 2) q^{N}\right) \\
& =-\frac{\pi^{2}}{3}\left(1-24 S_{1}+24 S_{2}\right) .
\end{aligned}
$$

(c) From (16), we have

$$
\begin{aligned}
\wp\left(\frac{1}{2}\right) & =\frac{2 \pi^{2}}{3}\left(\frac{\left(q^{2} ; q^{2}\right)_{\infty}^{20}}{(q)_{\infty}^{8}\left(q^{4} ; q^{4}\right)_{\infty}^{8}}-8 \frac{q\left(q^{4} ; q^{4}\right)_{\infty}^{8}}{\left(q^{2} ; q^{2}\right)_{\infty}^{4}}\right) \\
& =\frac{2 \pi^{2}}{3}\left(1+8 \sum_{N=1}^{\infty} q^{N}\left(2+(-1)^{N}\right) \sum_{\substack{\omega \mid N \\
\omega \text { odd }}} \omega-8 \sum_{N \text { odd }} \sigma(N) q^{N}\right) \\
& =\frac{2 \pi^{2}}{3}\left(1+8 \sum_{N \text { odd }} q^{N} \sum_{\substack{\omega \mid N \\
\omega \text { odd }}} \omega+24 \sum_{N \text { even }} q^{N} \sum_{\omega \mid N} \omega-8 \sum_{\omega \text { odd }} \sigma(N) q^{N}\right) \\
& =\frac{2 \pi^{2}}{3}\left(1+24 \sum_{N \text { oven }} q^{N} \sum_{\substack{\omega \mid N \\
\omega \text { odd }}} \omega\right)
\end{aligned}
$$




$$
\begin{aligned}
& =\frac{2 \pi^{2}}{3}\left(1+24 \sum_{N \text { even }} \sigma_{1,1}(N ; 2) q^{N}\right) \\
& =\frac{2 \pi^{2}}{3}\left(1+24 S_{2}\right) .
\end{aligned}
$$

This proves the lemma.

Using the fact that $e_{1}, e_{2}$ and $e_{3}$ are the roots of the equation $4 \wp(z)^{3}-g_{2}(\tau) \wp(z)-g_{3}(\tau)=$ 0 , indeed we can express $g_{2}(\tau)$ and $g_{3}(\tau)$ in terms of $S_{1}$ and $S_{2}$. More precisely, we have the following lemma.

Lemma 3.2 We have

$$
g_{2}(\tau)=\frac{4 \pi^{4}}{9}\left[3\left(1+24 S_{2}\right)^{2}+24^{2} S_{1}^{2}\right]
$$

and

$$
g_{3}(\tau)=\frac{8 \pi^{6}}{27}\left[\left(1+24 S_{2}\right)^{3}-24^{2} S_{1}^{2}\left(1+24 S_{2}\right)\right] .
$$

Proof Note that $g_{2}(\tau)=-4\left[e_{1} e_{2}+e_{2} e_{3}+e_{3} e_{1}\right]$, and hence

$$
\begin{aligned}
g_{2}(\tau)= & -4\left[\wp\left(\frac{\tau}{2}\right) \wp\left(\frac{1}{2}\right)+\wp\left(\frac{1}{2}\right) \wp\left(\frac{\tau+1}{2}\right)+\wp\left(\frac{\tau+1}{2}\right) \wp\left(\frac{\tau}{2}\right)\right] \\
= & -4\left[-\frac{2 \pi^{4}}{9}\left(1+24 S_{1}+24 S_{2}\right)\left(1+24 S_{2}\right)-\frac{2 \pi^{4}}{9}\left(1+24 S_{2}\right)\left(1-24 S_{1}+24 S_{2}\right)\right. \\
& \left.+\frac{\pi^{4}}{9}\left(1-24 S_{1}+24 S_{2}\right)\left(1+24 S_{1}+24 S_{2}\right)\right] \\
= & \frac{4 \pi^{4}}{9}\left[3\left(1+24 S_{2}\right)^{2}+24^{2} S_{1}^{2}\right] .
\end{aligned}
$$

Also note that $g_{3}(\tau)=4 e_{1} e_{2} e_{3}$, and hence

$$
\begin{aligned}
g_{3}(\tau) & =4 \wp\left(\frac{\tau}{2}\right) \wp\left(\frac{1}{2}\right) \wp\left(\frac{\tau+1}{2}\right) \\
& =\frac{8 \pi^{6}}{27}\left(1+24 S_{1}+24 S_{2}\right)\left(1+24 S_{2}\right)\left(1-24 S_{1}+24 S_{2}\right) \\
& =\frac{8 \pi^{6}}{27}\left[\left(1+24 S_{2}\right)^{3}-24^{2} S_{1}^{2}\left(1+24 S_{2}\right)\right] .
\end{aligned}
$$

This completes the proof of the lemma.

In the next theorem, we give $q$-series expressions for $E_{4}(\tau)$ and $E_{6}(\tau)$ with the coefficients involving restricted divisor functions $\sigma_{1,1}(N ; 2)$ and its convolution sums. Precisely, we prove the following theorem. 
Theorem 3.3 We have

$$
\begin{aligned}
E_{4}(\tau)= & \frac{2^{2} \cdot 3}{(2 \pi)^{4}} g_{2}(\tau) \\
= & 1+240 q+\sum_{M=2}^{\infty}\left[48 \sigma_{1,1}(2 M ; 2)+576 \sum_{\substack{k=1 \\
k+l=M}}^{M-1} \sigma_{1,1}(2 k ; 2) \sigma_{1,1}(2 l ; 2)\right. \\
& \left.+192 \sum_{\substack{k=1 \\
k+l-1=M}}^{M} \sigma_{1,1}(2 k-1 ; 2) \sigma_{1,1}(2 l-1 ; 2)\right] q^{M}
\end{aligned}
$$

and

$$
\begin{aligned}
E_{6}(\tau)= & \frac{2^{3} \cdot 3^{3}}{(2 \pi)^{6}} g_{3}(\tau) \\
= & 1-504 q-16,632 q^{2}+\sum_{M=3}^{\infty}\left[72 \sigma_{1,1}(2 M ; 2)+1,728 \sum_{\substack{k=1 \\
k+l=M}}^{M-1} \sigma_{1,1}(2 k ; 2) \sigma_{1,1}(2 l ; 2)\right. \\
& +13,824 \sum_{\substack{k=1 \\
k+l+m=M}}^{M-2} \sigma_{1,1}(2 k ; 2) \sigma_{1,1}(2 l ; 2) \sigma_{1,1}(2 m ; 2) \\
& -576 \sum_{\substack{k=1 \\
k+l-1=M}}^{M} \sigma_{1,1}(2 k-1 ; 2) \sigma_{1,1}(2 l-1 ; 2) \\
& \left.-13,824 \sum_{\substack{k=1 \\
k+l+m-1=M}}^{M-1} \sigma_{1,1}(2 k-1 ; 2) \sigma_{1,1}(2 l-1 ; 2) \sigma_{1,1}(2 m ; 2)\right] q^{M} .
\end{aligned}
$$

Proof From Lemma 3.2, we observe that

$$
\begin{aligned}
E_{4}(\tau)= & \frac{2^{2} \cdot 3}{(2 \pi)^{4}} g_{2}(\tau) \\
= & \frac{2^{2} \cdot 3}{(2 \pi)^{4}} \cdot \frac{4 \pi^{4}}{9}\left[3\left(1+24 S_{2}\right)^{2}+24^{2} S_{1}^{2}\right] \\
= & 1+48 S_{2}+576 S_{2}^{2}+192 S_{1}^{2} \\
= & 1+48 \sum_{M=1}^{\infty} \sigma_{1,1}(2 M ; 2) q^{2 M} \\
& +576 \sum_{M=2}^{\infty} \sum_{k=1}^{M-1} \sigma_{1,1}(2 k ; 2) \sigma_{1,1}(2 l ; 2) q^{2 M} \\
& +192 \sum_{\substack{M=1 \\
k+l=M}}^{\infty} \sum_{k=1}^{M} \sigma_{1,1}(2 k-1 ; 2) \sigma_{1,1}(2 l-1 ; 2) q^{2 M} \\
= & 1+48 \sigma_{1,1}(2 ; 2) q^{2}+48 \sum_{M=2}^{\infty} \sigma_{1,1}(2 M ; 2) q^{2 M}
\end{aligned}
$$




$$
\begin{aligned}
& +576 \sum_{M=2}^{\infty} \sum_{\substack{k=1 \\
k+l=M}}^{M-1} \sigma_{1,1}(2 k ; 2) \sigma_{1,1}(2 l ; 2) q^{2 M} \\
& +192 \sigma_{1,1}(1 ; 2) \sigma_{1,1}(1 ; 2) q^{2}+192 \sum_{\substack{M=2 \\
k+l-1=M}}^{\infty} \sum_{k=1}^{M} \sigma_{1,1}(2 k-1 ; 2) \sigma_{1,1}(2 l-1 ; 2) q^{2 M} \\
& =1+240 q^{2}+\sum_{M=2}^{\infty}\left[48 \sigma_{1,1}(2 M ; 2)+576 \sum_{\substack{k=1 \\
k+l=M}}^{M-1} \sigma_{1,1}(2 k ; 2) \sigma_{1,1}(2 l ; 2)\right. \\
& \left.+192 \sum_{\substack{k=1 \\
k+l-1=M}}^{M} \sigma_{1,1}(2 k-1 ; 2) \sigma_{1,1}(2 l-1 ; 2)\right] q^{2 M}
\end{aligned}
$$

and

$$
\begin{aligned}
& E_{6}(\tau)=\frac{2^{3} \cdot 3^{3}}{(2 \pi)^{6}} g_{3}(\tau) \\
& =1+72 S_{2}+1,728 S_{2}^{2}+13,824 S_{2}^{3}-576 S_{1}^{2}-13,824 S_{1}^{2} S_{2} \\
& =1+72 \sum_{M=1}^{\infty} \sigma_{1,1}(2 M ; 2) q^{2 M}+1,728 \sum_{\substack{M=2 \\
k+l=M}}^{\infty} \sum_{k=1}^{M-1} \sigma_{1,1}(2 k ; 2) \sigma_{1,1}(2 l ; 2) q^{2 M} \\
& +13,824 \sum_{\substack{M=3 \\
k+l+m=M}}^{\infty} \sum_{k=1}^{M-2} \sigma_{1,1}(2 k ; 2) \sigma_{1,1}(2 l ; 2) \sigma_{1,1}(2 m ; 2) q^{2 M} \\
& -576 \sum_{\substack{M=1 \\
k+l-1=M}}^{\infty} \sum_{k=1}^{M} \sigma_{1,1}(2 k-1 ; 2) \sigma_{1,1}(2 l-1 ; 2) q^{2 M} \\
& -13,824 \sum_{\substack{M=2 \\
k+l+m-1=M}}^{\infty} \sum_{k=1}^{M-1} \sigma_{1,1}(2 k-1 ; 2) \sigma_{1,1}(2 l-1 ; 2) \sigma_{1,1}(2 m ; 2) q^{2 M} \\
& =1+72 \sigma_{1,1}(2 ; 2) q^{2}+72 \sigma_{1,1}(4 ; 2) q^{4}+72 \sum_{M=3}^{\infty} \sigma_{1,1}(2 M ; 2) q^{2 M} \\
& +1,728 \sigma_{1,1}(2 ; 2) \sigma_{1,1}(2 ; 2) q^{4}+1,728 \sum_{M=3}^{\infty} \sum_{\substack{k=1 \\
k+l=M}}^{M-1} \sigma_{1,1}(2 k ; 2) \sigma_{1,1}(2 l ; 2) q^{2 M} \\
& +13,824 \sum_{M=3}^{\infty} \sum_{\substack{k=1 \\
k+l+m=M}}^{M-2} \sigma_{1,1}(2 k ; 2) \sigma_{1,1}(2 l ; 2) \sigma_{1,1}(2 m ; 2) q^{2 M} \\
& -576 \sigma_{1,1}(1 ; 2) \sigma_{1,1}(1 ; 2) q^{2}-576\left[\sigma_{1,1}(1 ; 2) \sigma_{1,1}(3 ; 2)+\sigma_{1,1}(3 ; 2) \sigma_{1,1}(1 ; 2)\right] \\
& -576 \sum_{M=3}^{\infty} \sum_{\substack{k=1 \\
k+l-1=M}}^{M} \sigma_{1,1}(2 k-1 ; 2) \sigma_{1,1}(2 l-1 ; 2) q^{2 M} \\
& -13,824 \sigma_{1,1}(1 ; 2) \sigma_{1,1}(1 ; 2) \sigma_{1,1}(2 ; 2) q^{4}
\end{aligned}
$$




$$
\begin{gathered}
-13,824 \sum_{M=3}^{\infty} \sum_{\substack{k=1 \\
k+l+m-1=M}}^{M-1} \sigma_{1,1}(2 k-1 ; 2) \sigma_{1,1}(2 l-1 ; 2) \sigma_{1,1}(2 m ; 2) q^{2 M} \\
=1+72 q^{2}+72 q^{4}+1,728 q^{4}-576 q^{2}-4,608 q^{4}-13,824 q^{4} \\
+\sum_{M=3}^{\infty}\left[72 \sigma_{1,1}(2 M ; 2)+1,728 \sum_{\substack{k=1 \\
k+l=M}}^{M-1} \sigma_{1,1}(2 k ; 2) \sigma_{1,1}(2 l ; 2)\right. \\
+13,824 \sum_{\substack{k=1 \\
k+l+m=M}}^{M-2} \sigma_{1,1}(2 k ; 2) \sigma_{1,1}(2 l ; 2) \sigma_{1,1}(2 m ; 2) \\
-576 \sum_{\substack{k=1 \\
k+l-1=M}}^{M} \sigma_{1,1}(2 k-1 ; 2) \sigma_{1,1}(2 l-1 ; 2) \\
\left.-13,824 \sum_{\substack{k=1 \\
k+l+m-1=M}}^{M-1} \sigma_{1,1}(2 k-1 ; 2) \sigma_{1,1}(2 l-1 ; 2) \sigma_{1,1}(2 m ; 2)\right] q^{2 M} .
\end{gathered}
$$

Now replacing $q^{2}$ into $q$ in the above expressions for $E_{4}(\tau)$ and $E_{6}(\tau)$, we obtain

$$
\begin{aligned}
E_{4}(\tau)= & +240 q+\sum_{M=2}^{\infty}\left[48 \sigma_{1,1}(2 M ; 2)+576 \sum_{\substack{k=1 \\
k+l=M}}^{M-1} \sigma_{1,1}(2 k ; 2) \sigma_{1,1}(2 l ; 2)\right. \\
& \left.+192 \sum_{\substack{k=1 \\
k+l-1=M}}^{M} \sigma_{1,1}(2 k-1 ; 2) \sigma_{1,1}(2 l-1 ; 2)\right] q^{M}
\end{aligned}
$$

and

$$
\begin{aligned}
E_{6}(\tau)= & 1-504 q-16,632 q^{2}+\sum_{M=3}^{\infty}\left[72 \sigma_{1,1}(2 M ; 2)+1,728 \sum_{\substack{k=1 \\
k+l=M}}^{M-1} \sigma_{1,1}(2 k ; 2) \sigma_{1,1}(2 l ; 2)\right. \\
& +13,824 \sum_{\substack{k=1 \\
k+l+m=M}}^{M-2} \sigma_{1,1}(2 k ; 2) \sigma_{1,1}(2 l ; 2) \sigma_{1,1}(2 m ; 2) \\
& -576 \sum_{\substack{k=1 \\
k+l-1=M}}^{M} \sigma_{1,1}(2 k-1 ; 2) \sigma_{1,1}(2 l-1 ; 2) \\
& \left.-13,824 \sum_{\substack{k=1 \\
k+l+m-1=M}}^{M-1} \sigma_{1,1}(2 k-1 ; 2) \sigma_{1,1}(2 l-1 ; 2) \sigma_{1,1}(2 m ; 2)\right] q^{M} .
\end{aligned}
$$

This completes the proof of the theorem.

It should be noted that $E_{4}(\tau)$ and $E_{6}(\tau)$ themselves have coefficients $\sigma_{3}(M)$ and $\sigma_{5}(M)$ in their $q$-series expansions. However, the aim of the next theorem is to express $\sigma_{3}(M)$ and $\sigma_{5}(M)$ in terms of convolution sums involving restricted divisor functions $\sigma_{1,1}(N ; 2)$. 
Theorem 3.4 (a) If $M \geq 2$ is an integer, then

$$
\begin{aligned}
5 \sigma_{3}(M)= & \sigma_{1,1}(M ; 2)+12 \sum_{k=1}^{M-1} \sigma_{1,1}(k ; 2) \sigma_{1,1}(M-k ; 2) \\
& +4 \sum_{k=1}^{M} \sigma_{1,1}(2 k-1 ; 2) \sigma_{1,1}(2 M-2 k+1 ; 2) .
\end{aligned}
$$

In particular, if $M \geq 3$ is odd, then

$$
\sum_{k=1}^{\frac{M-1}{2}} \sigma_{1,1}(k ; 2) \sigma_{1,1}(M-k ; 2)=\frac{1}{24}\left[\sigma_{3}(M)-\sigma_{1,1}(M ; 2)\right] .
$$

(b) If $M \geq 3$ is an integer, then

$$
\begin{aligned}
-7 \sigma_{5}(M)= & \sigma_{1,1}(M ; 2)+24 \sum_{k=1}^{M-1} \sigma_{1,1}(k ; 2) \sigma_{1,1}(M-k ; 2) \\
& +192 \sum_{k, l=1}^{M-2} \sigma_{1,1}(k ; 2) \sigma_{1,1}(l ; 2) \sigma_{1,1}(M-k-l ; 2) \\
& -8 \sum_{k=1}^{M} \sigma_{1,1}(2 k-1 ; 2) \sigma_{1,1}(2 M-2 k+1 ; 2) \\
& -192 \sum_{k, l=1}^{M-1} \sigma_{1,1}(2 k-1 ; 2) \sigma_{1,1}(2 l-1 ; 2) \sigma_{1,1}(M-k-l+1 ; 2)
\end{aligned}
$$

In particular, if $M \geq 3$ is odd, then

$$
\begin{aligned}
-7 \sigma_{5}(M)+8 \sigma_{3}(M)= & \sigma_{1,1}(M ; 2)+24 \sum_{k=1}^{M-1} \sigma_{1,1}(k ; 2) \sigma_{1,1}(M-k ; 2) \\
& +192 \sum_{k, l=1}^{M-2} \sigma_{1,1}(k ; 2) \sigma_{1,1}(l ; 2) \sigma_{1,1}(M-k-l ; 2) \\
& -192 \sum_{k, l=1}^{M-1} \sigma_{1,1}(2 k-1 ; 2) \sigma_{1,1}(2 l-1 ; 2) \sigma_{1,1}(M-k-l+1 ; 2) .
\end{aligned}
$$

Proof From [19, p.59], we know that

$$
E_{4}(\tau)=1+240 \sum_{M \geq 1} \sigma_{3}(M) q^{M}
$$

and

$$
E_{6}(\tau)=1-504 \sum_{M \geq 1} \sigma_{5}(M) q^{M}
$$


So comparing (24) and (26), we find that

$$
\begin{aligned}
5 \sigma_{3}(M)= & \sigma_{1,1}(2 M ; 2)+12 \sum_{\substack{k=1 \\
k+l=M}}^{M-1} \sigma_{1,1}(2 k ; 2) \sigma_{1,1}(2 l ; 2) \\
& +4 \sum_{\substack{k=1 \\
k+l-1=M}}^{M} \sigma_{1,1}(2 k-1 ; 2) \sigma_{1,1}(2 l-1 ; 2) \\
= & \sigma_{1,1}(M ; 2)+12 \sum_{k=1}^{M-1} \sigma_{1,1}(k ; 2) \sigma_{1,1}(M-k ; 2) \\
& +4 \sum_{k=1}^{M} \sigma_{1,1}(2 k-1 ; 2) \sigma_{1,1}(2 M-2 k+1 ; 2),
\end{aligned}
$$

where $M \geq 2$.

On the other hand, Liouville [25] proved

$$
\sigma_{3}(M)=\sum_{k=1}^{M} \sigma(2 k-1) \sigma(2 M-2 k+1)
$$

for odd $M$. From (28) and (29), we note that we reprove a result in [26, p.300], namely,

$$
5 \sigma_{3}(M)=\sigma_{1,1}(M ; 2)+12 \sum_{k=1}^{M-1} \sigma_{1,1}(k ; 2) \sigma_{1,1}(M-k ; 2)+4 \sigma_{3}(M)
$$

and so,

$$
\begin{aligned}
\sigma_{3}(M) & =\sigma_{1,1}(M ; 2)+12 \sum_{k=1}^{M-1} \sigma_{1,1}(k ; 2) \sigma_{1,1}(M-k ; 2) \\
& =\sigma(M)+24 \sum_{k=1}^{\frac{M-1}{2}} \sigma_{1,1}(k ; 2) \sigma_{1,1}(M-k ; 2)
\end{aligned}
$$

for odd $M$. By the same way, comparing (25) and (27), we deduce

$$
\begin{aligned}
-7 \sigma_{5}(M)= & \sigma_{1,1}(2 M ; 2)+24 \sum_{\substack{k=1 \\
k+l=M}}^{M-1} \sigma_{1,1}(2 k ; 2) \sigma_{1,1}(2 l ; 2) \\
& +192 \sum_{\substack{k=1 \\
k+l+m=M}}^{M-2} \sigma_{1,1}(2 k ; 2) \sigma_{1,1}(2 l ; 2) \sigma_{1,1}(2 m ; 2) \\
& -8 \sum_{\substack{k=1 \\
k+l-1=M}}^{M} \sigma_{1,1}(2 k-1 ; 2) \sigma_{1,1}(2 l-1 ; 2) \\
& -192 \sum_{\substack{k=1 \\
k+l+m-1=M}}^{M-1} \sigma_{1,1}(2 k-1 ; 2) \sigma_{1,1}(2 l-1 ; 2) \sigma_{1,1}(2 m ; 2)
\end{aligned}
$$




$$
\begin{aligned}
= & \sigma_{1,1}(M ; 2)+24 \sum_{k=1}^{M-1} \sigma_{1,1}(k ; 2) \sigma_{1,1}(M-k ; 2) \\
& +192 \sum_{k, l=1}^{M-2} \sigma_{1,1}(k ; 2) \sigma_{1,1}(l ; 2) \sigma_{1,1}(M-k-l ; 2) \\
& -8 \sum_{k=1}^{M} \sigma_{1,1}(2 k-1 ; 2) \sigma_{1,1}(2 M-2 k+1 ; 2) \\
& -192 \sum_{k, l=1}^{M-1} \sigma_{1,1}(2 k-1 ; 2) \sigma_{1,1}(2 l-1 ; 2) \sigma_{1,1}(M-k-l+1 ; 2),
\end{aligned}
$$

where $M \geq 3$. Note that for odd $M \geq 3$, we get

$$
\begin{aligned}
-7 \sigma_{5}(M)= & \sigma_{1,1}(M ; 2)+24 \sum_{k=1}^{M-1} \sigma_{1,1}(k ; 2) \sigma_{1,1}(M-k ; 2) \\
& +192 \sum_{k, l=1}^{M-2} \sigma_{1,1}(k ; 2) \sigma_{1,1}(l ; 2) \sigma_{1,1}(M-k-l ; 2)-8 \sigma_{3}(M) \\
& -192 \sum_{k, l=1}^{M-1} \sigma_{1,1}(2 k-1 ; 2) \sigma_{1,1}(2 l-1 ; 2) \sigma_{1,1}(M-k-l+1 ; 2),
\end{aligned}
$$

where we used Equation (29). This completes the proof of the theorem.

\section{Proof of the theorems}

Proof of Theorem 1.1 Glaisher proved that (see [26, p.300])

$$
\sigma(1) \sigma(2 n-1)+\sigma(3) \sigma(2 n-3)+\cdots+\sigma(2 n-1) \sigma(1)=\frac{1}{8}\left[\sigma_{3}(2 n)-\sigma_{3}(n)\right] .
$$

It follows directly from (28) that

$$
\begin{gathered}
\sigma(1) \sigma(2 M-1)+\sigma(3) \sigma(2 M-3)+\cdots+\sigma(2 M-1) \sigma(1) \\
\quad=\sum_{k=1}^{M} \sigma_{1,1}(2 k-1 ; 2) \sigma_{1,1}(2 M-2 k+1 ; 2)
\end{gathered}
$$

and

$$
5 \sigma_{3}(M)=\sigma_{1,1}(M ; 2)+12 \sum_{k=1}^{M-1} \sigma_{1,1}(k ; 2) \sigma_{1,1}(M-k ; 2)+\frac{1}{2}\left[\sigma_{3}(2 M)-\sigma_{3}(M)\right] .
$$

Thus, we have proved the identity

$$
\sum_{k=1}^{M-1} \sigma_{1,1}(k ; 2) \sigma_{1,1}(M-k ; 2)=\frac{1}{24}\left[11 \sigma_{3}(M)-\sigma_{3}(2 M)-2 \sigma_{1,1}(M ; 2)\right] .
$$


Thus, for any integer $M \geq 2$, we have

$$
11 \sigma_{3}(M) \equiv \sigma_{3}(2 M)+2 \sigma_{1,1}(M ; 2) \quad(\bmod 24)
$$

This proves (i).

We note that we can write (34) as

$$
\begin{aligned}
& 2 \sum_{k<\frac{M}{2}} \sigma_{1,1}(k ; 2) \sigma_{1,1}(M-k ; 2)+\sigma_{1,1}\left(\frac{M}{2} ; 2\right) \sigma_{1,1}\left(\frac{M}{2} ; 2\right) \\
& \quad=\frac{1}{24}\left[11 \sigma_{3}(M)-\sigma_{3}(2 M)-2 \sigma_{1,1}(M ; 2)\right] .
\end{aligned}
$$

If $M$ is odd, then (35) is equivalent to

$$
24 \cdot 2 \sum_{k<\frac{M}{2}} \sigma_{1,1}(k ; 2) \sigma_{1,1}(M-k ; 2)=11 \sigma_{3}(M)-\sigma_{3}(2 M)-2 \sigma_{1,1}(M ; 2)
$$

and hence,

$$
11 \sigma_{3}(M) \equiv \sigma_{3}(2 M)+2 \sigma_{1,1}(M ; 2) \quad(\bmod 48)
$$

Let $M=2^{m} p^{n} p_{1}^{e_{1}} p_{2}^{e_{2}} \cdots p_{r}^{e_{r}}$. Therefore, if $\operatorname{ord}_{p} M=n$ is odd for an odd prime $p$, then we note that

$$
\begin{aligned}
\sigma_{1,1}\left(\frac{M}{2} ; 2\right) \sigma_{1,1}\left(\frac{M}{2} ; 2\right) & =\sigma_{1,1}\left(2^{m-1} p^{n} p_{1}^{e_{1}} p_{2}^{e_{2}} \cdots p_{r}^{e_{r}} ; 2\right) \sigma_{1,1}\left(2^{m-1} p^{n} p_{1}^{e_{1}} p_{2}^{e_{2}} \cdots p_{r}^{e_{r}} ; 2\right) \\
& =\left\{\sigma_{1,1}\left(2^{m-1} ; 2\right)\right\}^{2}\left\{\sigma_{1}\left(p^{n} p_{1}^{e_{1}} p_{2}^{e_{2}} \cdots p_{r}^{e_{r}}\right)\right\}^{2} \\
& =\left\{\sigma_{1}\left(p^{n}\right)\right\}^{2}\left\{\sigma_{1,1}\left(2^{m-1} ; 2\right)\right\}^{2}\left\{\sigma_{1}\left(p_{1}^{e_{1}} p_{2}^{e_{2}} \cdots p_{r}^{e_{r}}\right)\right\}^{2} \\
& =(2 l)^{2}\left\{\sigma_{1,1}\left(2^{m-1} ; 2\right)\right\}^{2}\left\{\sigma_{1}\left(p_{1}^{e_{1}} p_{2}^{e_{2}} \cdots p_{r}^{e_{r}}\right)\right\}^{2},
\end{aligned}
$$

since

$$
\sigma_{1}\left(p^{n}\right)=1+p+p^{2}+\cdots+p^{n} \equiv 0 \quad(\bmod 2)
$$

and so we can write $\sigma_{1}\left(p^{n}\right)=2 l$ for some $l \in \mathbb{N}$. Therefore, from (35), we obtain

$$
\begin{aligned}
& 2 \sum_{k<\frac{M}{2}} \sigma_{1,1}(k ; 2) \sigma_{1,1}(M-k ; 2)+2 \cdot 2 l^{2}\left\{\sigma_{1,1}\left(2^{m-1} ; 2\right)\right\}^{2}\left\{\sigma_{1}\left(p_{1}^{e_{1}} p_{2}^{e_{2}} \cdots p_{r}^{e_{r}}\right)\right\}^{2} \\
& \quad=\frac{1}{24}\left[11 \sigma_{3}(M)-\sigma_{3}(2 M)-2 \sigma_{1,1}(M ; 2)\right] .
\end{aligned}
$$

This means that

$$
11 \sigma_{3}(M) \equiv \sigma_{3}(2 M)+2 \sigma_{1,1}(M ; 2) \quad(\bmod 48)
$$

This proves (ii). 
Remark 4.1 Define

$$
P(N):=\{p: p \mid N, p \text { is a positive prime }\}
$$

and

$$
P_{\max }(N):=\max _{p \in P(N)} p .
$$

For example, $P(10)=\{2,5\}$ and $P_{\max }(10)=5$. For any prime $p \geq 5$, we note that

$$
P_{\max }\left(\sum_{k=1}^{p-1} \sigma_{1,1}(k ; 2) \sigma_{1,1}(p-k ; 2)\right)=p
$$

We observe that

$$
\sum_{k=1}^{p-1} \sigma_{1,1}(k ; 2) \sigma_{1,1}(p-k ; 2)=\frac{1}{2}\left(\begin{array}{c}
p+1 \\
3
\end{array}\right)=\frac{(p+1) p(p-1)}{12}
$$

and $(p, 12)=1$. Thus, $12 \mid\left(p^{2}-1\right)$ and this implies that the largest prime factor of $p^{2}-1$ is strictly $<p$.

Remark 4.2 From the fact

$$
\sigma_{1,1}(k ; 2)=\sigma_{1}(k)-2 \sigma_{1}\left(\frac{k}{2}\right)
$$

and from known results, it is possible to establish the following identity (in an elementary way, without using the Eisenstein series), namely

$$
\sum_{k=1}^{M-1} \sigma_{1,1}(k ; 2) \sigma_{1,1}(M-k ; 2)=\frac{1}{12} \sigma_{3}(M)+\frac{1}{3} \sigma_{3}\left(\frac{M}{2}\right)-\frac{1}{12} \sigma_{1}(M)+\frac{1}{6} \sigma_{1}\left(\frac{M}{2}\right) .
$$

One can also use this identity to obtain

$$
\sum_{k=1}^{p-1} \sigma_{1,1}(k ; 2) \sigma_{1,1}(p-k ; 2)=\frac{1}{2}\left(\begin{array}{c}
p+1 \\
3
\end{array}\right) .
$$

Proof of Theorem 1.2 Inserting (33) and (34) into (31), we obtain

$$
\begin{aligned}
-7 \sigma_{5}(M)= & \sigma_{1,1}(M ; 2)+24 \cdot \frac{1}{24}\left[11 \sigma_{3}(M)-\sigma_{3}(2 M)-2 \sigma_{1,1}(M ; 2)\right] \\
& +192 \sum_{k, l=1}^{M-2} \sigma_{1,1}(k ; 2) \sigma_{1,1}(l ; 2) \sigma_{1,1}(M-k-l ; 2)-8 \cdot \frac{1}{8}\left[\sigma_{3}(2 M)-\sigma_{3}(M)\right] \\
& -192 \sum_{k, l=1}^{M-1} \sigma_{1,1}(2 k-1 ; 2) \sigma_{1,1}(2 l-1 ; 2) \sigma_{1,1}(M-k-l+1 ; 2) .
\end{aligned}
$$


Thus, we deduce the congruence relation

$$
-7 \sigma_{5}(M)+\sigma_{1,1}(M ; 2) \equiv 12 \sigma_{3}(M)-2 \sigma_{3}(2 M) \quad(\bmod 192) .
$$

This completes the proof of Theorem 1.2.

Proof of Theorem 1.3 We expand $S_{1}^{3}$ as

$$
\begin{aligned}
& \sum_{n, l, m=1}^{\infty} \sigma_{1,1}(2 n-1 ; 2) \sigma_{1,1}(2 l-1 ; 2) \sigma_{1,1}(2 m-1 ; 2) q^{2(n+l+m-1)-1} \\
& =\sum_{N=2}^{\infty}\left\{\sum_{M=1}^{N-1} \sum_{m=1}^{M} \sigma_{1,1}(2 N-1-2 M ; 2)\right. \\
& \left.\quad \times \sigma_{1,1}(2 M-(2 m-1) ; 2) \sigma_{1,1}(2 m-1 ; 2)\right\} q^{2 N-1}
\end{aligned}
$$

Then we notice that

$$
\begin{aligned}
& \sum_{M=1}^{N-1} \sum_{m=1}^{M} \sigma_{1,1}(2 N-1-2 M ; 2) \sigma_{1,1}(2 M-(2 m-1) ; 2) \sigma_{1,1}(2 m-1 ; 2) \\
& \quad=\sum_{M=1}^{N-1} \sigma_{1,1}(2 N-1-2 M ; 2)\left\{\sum_{m=1}^{M} \sigma_{1,1}(2 M-(2 m-1) ; 2) \sigma_{1,1}(2 m-1 ; 2)\right\} \\
& \quad=\sum_{M=1}^{N-1} \sigma_{1}(2 N-1-2 M) \cdot \frac{1}{8}\left(\sigma_{3}(2 M)-\sigma_{3}(M)\right)
\end{aligned}
$$

by [27, Lemma 3.1(b)] and $\sigma_{1,1}($ odd 2$)=\sigma_{1}($ odd $)$. Since $\sigma_{3}(2 M)=9 \sigma_{3}(M)-8 \sigma_{3}\left(\frac{M}{2}\right)$, the right-hand side of (36) is

$$
\begin{aligned}
= & \sum_{M=1}^{N-1} \sigma_{1}(2 N-1-2 M)\left\{\sigma_{3}(M)-\sigma_{3}\left(\frac{M}{2}\right)\right\} \\
= & \sum_{M=1}^{N-1} \sigma_{1}(2 N-1-2 M) \sigma_{3}(M)-\sum_{M<\frac{2 N-1}{4}} \sigma_{1}(2 N-1-4 M) \sigma_{3}(M) \\
= & \frac{1}{240}\left(\sigma_{5}(2 N-1)-\sigma_{1}(2 N-1)\right) \\
& -\left\{\frac{1}{3,840} \sigma_{5}(2 N-1)-\frac{1}{240} \sigma_{1}(2 N-1)+\frac{1}{256} a(2 N-1)\right\}
\end{aligned}
$$

by $[25$, Theorem 6$]$ and in $[28$, Theorem 4.1 (iii)] which state that

$$
\begin{aligned}
& \sum_{m<N / 2} \sigma_{3}(m) \sigma_{1}(N-2 m) \\
& \quad=\frac{1}{240}\left(\sigma_{5}(N)-\sigma(N)+20 \sigma_{5}\left(\frac{N}{2}\right)+(10-30 N) \sigma_{3}\left(\frac{N}{2}\right)\right)
\end{aligned}
$$


and

$$
\begin{aligned}
\sum_{m<N / 4} \sigma_{3}(m) \sigma_{1}(N-4 m)= & \frac{1}{3,840} \sigma_{5}(N)+\frac{1}{256} \sigma_{5}\left(\frac{N}{2}\right)+\frac{1}{12} \sigma_{5}\left(\frac{N}{4}\right) \\
& +\frac{1-3 N}{24} \sigma_{3}\left(\frac{N}{4}\right)-\frac{1}{240} \sigma_{1}(N)+\frac{1}{256} a(N)
\end{aligned}
$$

respectively. This completes the proof.

Proof of Corollary 1.4 We note that

$$
\begin{aligned}
& \sum_{\substack{n+l+m-1=N \\
n, l, m \geq 1}}(2 n-1) \sigma_{1,1}(2 n-1 ; 2) \sigma_{1,1}(2 l-1 ; 2) \sigma_{1,1}(2 m-1 ; 2) \\
& =\frac{1}{3} \sum_{\substack{n+l+m-1=N \\
n, l, m \geq 1}}(2(n+l+m-1)-1) \sigma_{1,1}(2 n-1 ; 2) \sigma_{1,1}(2 l-1 ; 2) \sigma_{1,1}(2 m-1 ; 2) \\
& =\frac{1}{3} \sum_{\substack{n+l+m-1=N \\
n, l, m \geq 1}}(2 N-1) \sigma_{1,1}(2 n-1 ; 2) \sigma_{1,1}(2 l-1 ; 2) \sigma_{1,1}(2 m-1 ; 2) .
\end{aligned}
$$

Therefore, from Theorem 1.3, we obtain

$$
\begin{aligned}
& \sum_{\substack{n+l+m-1=N \\
n, l, m \geq 1}} n \sigma_{1,1}(2 n-1 ; 2) \sigma_{1,1}(2 l-1 ; 2) \sigma_{1,1}(2 m-1 ; 2) \\
& =\frac{N+1}{3} \sum_{\substack{n+l+m-1=N \\
n, l, m \geq 1}} \sigma_{1,1}(2 n-1 ; 2) \sigma_{1,1}(2 l-1 ; 2) \sigma_{1,1}(2 m-1 ; 2) \\
& =\frac{N+1}{768}\left(\sigma_{5}(2 N-1)-a(2 N-1)\right) .
\end{aligned}
$$

Thus, the first assertion

$$
(N+1) a(2 N-1) \equiv(N+1) \sigma_{5}(2 N-1) \quad(\bmod 768)
$$

follows.

We note that $768=2^{8} \cdot 3$, and thus whenever $N+1 \not \equiv 0(\bmod 3)$ and $N+1$ is odd, we have $(N+1,768)=1$. Thus,

$$
\sigma_{5}(2 N-1) \equiv a(2 N-1) \quad(\bmod 768)
$$

whenever $N \equiv 0,4(\bmod 6)$. This proves the second assertion.

Proof of Theorem 1.5 We note that

$$
\begin{aligned}
S_{1} S_{2}^{2} & =\sum_{n, l, m=1}^{\infty} \sigma_{1,1}(2 n-1 ; 2) \sigma_{1,1}(2 l ; 2) \sigma_{1,1}(2 m ; 2) q^{2(n+l+m)-1} \\
& =\sum_{N=3}^{\infty}\left\{\sum_{M=2}^{N-1} \sum_{m=1}^{M-1} \sigma_{1,1}(2 N-1-2 M ; 2) \sigma_{1,1}(2 M-2 m ; 2) \sigma_{1,1}(2 m ; 2)\right\} q^{2 N-1} .
\end{aligned}
$$


Thus, by $\sigma_{1,1}(2 m ; 2)=\sigma_{1,1}(m ; 2)$ and $[27,(11)]$, we obtain that

$$
\begin{aligned}
& \sum_{M=2}^{N-1} \sum_{m=1}^{M-1} \sigma_{1,1}(2 N-1-2 M ; 2) \sigma_{1,1}(2 M-2 m ; 2) \sigma_{1,1}(2 m ; 2) \\
& \quad=\sum_{M=2}^{N-1} \sigma_{1,1}(2 N-1-2 M ; 2)\left\{\sum_{m=1}^{M-1} \sigma_{1,1}(M-m ; 2) \sigma_{1,1}(m ; 2)\right\} \\
& =\sum_{M=2}^{N-1} \sigma_{1}(2 N-1-2 M)\left\{\frac{1}{24}\left(11 \sigma_{3}(M)-\sigma_{3}(2 M)-2 \sigma_{1,1}(M ; 2)\right)\right\} .
\end{aligned}
$$

We observe that

$$
\begin{aligned}
& 11 \sigma_{3}(M)-\sigma_{3}(2 M)-2 \sigma_{1,1}(M ; 2) \\
& \quad=11 \sigma_{3}(M)-\left\{9 \sigma_{3}(M)-8 \sigma_{3}\left(\frac{M}{2}\right)\right\}-2\left\{\sigma_{1}(M)-2 \sigma_{1}\left(\frac{M}{2}\right)\right\} \\
& =2 \sigma_{3}(M)+8 \sigma_{3}\left(\frac{M}{2}\right)-2 \sigma_{1}(M)+4 \sigma_{1}\left(\frac{M}{2}\right) .
\end{aligned}
$$

Thus, the right-hand side of (39) is

$$
\begin{aligned}
= & \frac{1}{24}\left[2 \sum_{M=1}^{N-1} \sigma_{1}(2 N-1-2 M) \sigma_{3}(M)+8 \sum_{M<\frac{2 N-1}{4}} \sigma_{1}(2 N-1-4 M) \sigma_{3}(M)\right. \\
& \left.-2 \sum_{M=1}^{N-1} \sigma_{1}(2 N-1-2 M) \sigma_{1}(M)+4 \sum_{M<\frac{2 N-1}{4}} \sigma_{1}(2 N-1-4 M) \sigma_{1}(M)\right] .
\end{aligned}
$$

Now from (37), (38) and (see [25, (4.4)]), we have

$$
\begin{aligned}
& \sum_{m<N / 2} \sigma(m) \sigma(N-2 m) \\
& \quad=\frac{1}{24}\left(2 \sigma_{3}(N)+(1-3 N) \sigma_{1}(N)+8 \sigma_{3}\left(\frac{N}{2}\right)+(1-6 N) \sigma\left(\frac{N}{2}\right)\right)
\end{aligned}
$$

and from (see [25, Theorem 4]), we have

$$
\begin{aligned}
& \sum_{m<N / 4} \sigma(m) \sigma(N-4 m) \\
& \quad=\frac{1}{48}\left(\sigma_{3}(N)+(2-3 N) \sigma_{1}(N)+3 \sigma_{3}\left(\frac{N}{2}\right)+16 \sigma_{3}\left(\frac{N}{4}\right)+(2-12 N) \sigma_{1}\left(\frac{N}{4}\right)\right) .
\end{aligned}
$$

Thus, the theorem follows. 


\section{Author details}

${ }^{1}$ National Institute for Mathematical Sciences, Yuseong-daero 1689-gil, Yuseong-gu, Daejeon, 305-811, South Korea. ${ }^{2}$ Department of Mathematics and Institute of Pure and Applied Mathematics, Chonbuk National University, Chonju, Chonbuk 561-756, South Korea. ${ }^{3}$ Current address: National Institute for Mathematical Sciences, Yuseong-daero 1689-gil, Yuseong-gu, Daejeon, 305-811, South Korea. ${ }^{4}$ Permanent address: School of Mathematics, Tata Institute of Fundamental Research, Homi Bhabha Road, Mumbai, 400005, India.

\section{Acknowledgements}

Dedicated to Professor Hari M Srivastava.

The author, Ayyadurai Sankaranarayanan, wishes to thank the National Institute for Mathematical Sciences (NIMS), Daejeon, Republic of Korea, for its warm hospitality and generous support. This research was supported by the National Institute for Mathematical Sciences (NIMS) grant funded by the Korean government (B21303).

\section{Received: 17 January 2013 Accepted: 20 March 2013 Published: 2 April 2013}

\section{References}

1. Ramanujan, S: Collected Papers of Srinivasa Ramanujan. AMS Chelsea Publishing, Providence (2000)

2. Glaisher, JWL: Expressions for the first five powers of the series in which the coefficients are sums of the divisors of the exponents. Messenger Math. 15, 33-36 (1885)

3. Lahiri, DB: On Ramanujan's function $\tau(n)$ and the divisor function $\sigma_{1}(n)$ - I. Bull. Calcutta Math. Soc. 38, 193-206 (1946)

4. Williams, KS: Number Theory in the Spirit of Liouville. London Mathematical Society Student Texts, vol. 76. Cambridge University Press, Cambridge (2011)

5. Berndt, BC: Ramanujan's Notebooks. Part II. Springer, New York (1989)

6. Berndt, BC, Evans, RJ: Chapter 15 of Ramanujan's second notebook, part II. Modular forms. Acta Arith. 47, 123-142 (1986)

7. Ramamani, V: On some identities conjectured by Srinivasa Ramanujan found in his lithographed notes connected with partition theory and elliptic modular functions. PhD thesis, University of Mysore (1970)

8. Lahiri, DB: On Ramanujan's function $\tau(n)$ and the divisor function $\sigma_{k}(n)$ - II. Bull. Calcutta Math. Soc. 39, 33-52 (1947)

9. Ono, K, Robins, S, Wahl, DT: On the representation of integer as sum of triangular numbers. Aequ. Math. 50, 73-94 (1995)

10. Williams, KS: On Liouville's twelve squares theorem. Far East J. Math. Sci. 29, 239-242 (2008)

11. Williams, KS: The convolution sum $\sum_{m<\frac{n}{8}} \sigma_{1}(m) \sigma_{1}(n-8 m)$. Pac. J. Math. 228, 387-396 (2006)

12. Chang, $\mathrm{C}-\mathrm{H}$, Srivastava, $\mathrm{HM}$ : A note on Bernoulli identities associated with the Weierstrass $\wp$-function. Integral Transforms Spec. Funct. 18, 245-253 (2007)

13. Chang, C-H, Srivastava, HM, Wu, T-C: Some families of Weierstrass-type functions and their applications. Integral Transforms Spec. Funct. 19, 621-632 (2008)

14. Aygunes, AA, Simsek, Y: The action of Hecke operators to families of Weierstrass-type functions and Weber-type functions and their applications. Appl. Math. Comput. 218, 678-682 (2011)

15. Melfi, G: On some modular identities. In: Number Theory, pp. 371-382. de Gruyter, Berlin (1998)

16. Dickson, LE: History of the Theory of Numbers, vol. II. Chelsea, New York (1952)

17. Ramanujan, S: On certain arithmetical functions. Trans. Camb. Philos. Soc. 22, 159-184 (1916)

18. Kim, D, Koo, JK: Algebraic integer as values of elliptic functions. Acta Arith. 100, 105-116 (2001)

19. Silverman, JH: Advanced Topics in the Arithmetic of Elliptic Curves. Springer, Berlin (1994)

20. Fine, NJ: Basic Hypergeometric Series and Applications. Am. Math. Soc., Providence (1988)

21. Cho, B, Kim, D, Koo, JK: Divisor functions arising from q-series. Publ. Math. (Debr.) 76, 495-508 (2010)

22. Cho, B, Kim, D, Koo, JK: Modular forms arising from divisor functions. J. Math. Anal. Appl. 356, 537-547 (2009)

23. Srivastava, HM: Some generalizations and basic (or $q-$ ) extensions of the Bernoulli, Euler and Genocchi polynomials. Appl. Math. Inf. Sci. 5, 390-444 (2011)

24. Srivastava, HM, Choi, J: Zeta and q-Zeta Functions and Associated Series and Integrals. Elsevier, Amsterdam (2012)

25. Huard, JG, Ou, ZM, Spearman, BK, Williams, KS: Elementary evaluation of certain convolution sums involving divisor functions. In: Number Theory for the Millennium, vol. II, pp. 229-274 (2000)

26. Dickson, LE: History of the Theory of Numbers, vol. I. Chelsea, New York (1952)

27. Kim, D, Kim, A, Li, Y: Convolution sums arising from the divisor functions. J. Korean Math. Soc. (to appear)

28. Cheng, N, Williams, KS: Evaluation of some convolution sums involving the sum of divisors functions. Yokohama Math. J. 52, 39-57 (2005) 